\title{
Spectral sensitivity, spatial resolution and temporal resolution and their implications for conspecific signalling in cleaner shrimp
}

\author{
Eleanor M. Caves ${ }^{1, *}$, Tamara M. Frank ${ }^{2}$ and Sönke Johnsen ${ }^{1}$
}

\begin{abstract}
Cleaner shrimp (Decapoda) regularly interact with conspecifics and client reef fish, both of which appear colourful and finely patterned to human observers. However, whether cleaner shrimp can perceive the colour patterns of conspecifics and clients is unknown, because cleaner shrimp visual capabilities are unstudied. We quantified spectral sensitivity and temporal resolution using electroretinography (ERG), and spatial resolution using both morphological (inter-ommatidial angle) and behavioural (optomotor) methods in three cleaner shrimp species: Lysmata amboinensis, Ancylomenes pedersoni and Urocaridella antonbruunii. In all three species, we found strong evidence for only a single spectral sensitivity peak of (mean \pm s.e.m.) $518 \pm 5,518$ \pm 2 and $533 \pm 3 \mathrm{~nm}$, respectively. Temporal resolution in darkadapted eyes was $39 \pm 1.3,36 \pm 0.6$ and $34 \pm 1.3 \mathrm{~Hz}$. Spatial resolution was $9.9 \pm 0.3,8.3 \pm 0.1$ and $11 \pm 0.5 \mathrm{deg}$, respectively, which is low compared with other compound eyes of similar size. Assuming monochromacy, we present approximations of cleaner shrimp perception of both conspecifics and clients, and show that cleaner shrimp visual capabilities are sufficient to detect the outlines of large stimuli, but not to detect the colour patterns of conspecifics or clients, even over short distances. Thus, conspecific viewers have probably not played a role in the evolution of cleaner shrimp appearance; rather, further studies should investigate whether cleaner shrimp colour patterns have evolved to be viewed by client reef fish, many of which possess triand tetra-chromatic colour vision and relatively high spatial acuity.
\end{abstract}

KEY WORDS: Visual signals, Decapod crustaceans, Colour patterns, Perception, Crustacean vision

\section{INTRODUCTION}

Animal visual capabilities are diverse, and understanding how an organism perceives visual scenes requires knowledge of that animal's spectral sensitivity (wavelength sensitivity of the photoreceptors), temporal resolution (ability to visually track rapid changes in a scene; Frank, 1999, 2003) and spatial resolution (ability to perceive detail). Numerous studies have shown that it is inaccurate to use human perception of colour and luminance to assess how an animal perceives a visual scene (Bennett et al., 1994; Safran and Vitousek, 2008); however, studies that quantify and incorporate receiver-appropriate measures of temporal

\footnotetext{
Department of Biology, Box 90338, Duke University, Durham, NC 27708, USA. ${ }^{2}$ Halmos College of Natural Sciences and Oceanography, Department of Marine and Environmental Sciences, Nova Southeast University, 8000 North Ocean Drive, Dania Beach, FL 33004, USA.

*Author for correspondence (emc39@duke.edu)
}

Received 13 March 2015; Accepted 3 December 2015 or spatial resolution are rare (but see Baldwin and Johnsen, 2011; Johnsen, 2006; Marshall, 2000).

Broadly, visual physiology may be adapted for certain tasks, such as the detection of relevant visual signals (summarized in Cronin et al., 2014; Land and Nilsson, 2002). For example, the multiple chromatic channels in some primates assist in foraging for fruit against a leafy background (Osorio and Vorobyev, 1996; Regan et al., 2001), and 'acute zones' in the eyes of Polistes paper wasps allow individuals to resolve the facial patterns of conspecifics (Sheehan et al., 2014). However, existing work on the relationship between visual capabilities and visual signals has focused on how a single aspect of vision relates to the detection of a signal. In reality, multiple aspects of an animal's visual physiology, including spectral sensitivity and both temporal and spatial resolution, all play a role in whether or not an animal can detect a given signal.

The interactions of cleaner shrimp with client reef fish are well described (reviewed in Côté, 2000; Losey et al., 1999a), but their visual ecology is poorly understood. Cleaner shrimp attract clients to cleaning stations, which they usually occupy in groups or pairs (Bauer, 2006; Briones-Fourzán et al., 2012; Bshary et al., 2007; Chapuis and Bshary, 2010; Fletcher et al., 1995; Huebner and Chadwick, 2012b), and then provide cleaning services to clients by removing ectoparasites, in turn receiving a meal. Thus, cleaner shrimp interact on a regular basis with conspecifics and client fish, both of which, to human observers, display brilliant colours and fine patterns. Evidence from the cleaner shrimps Lysmata debelius and Lysmata amboinensis suggests that the formation and maintenance of conspecific groups relies at least in part on visual cues (Fletcher et al., 1995; Rufino and Jones, 2001; Simoes and Jones, 1999), which could include colour patterns as an intraspecific signal. Additionally, to initiate cleaning, clients approach cleaners and adopt a characteristic solicitation pose, a stationary position with opercula and/or fins flared out, occasionally accompanied by a colour change (Côté et al., 1998; Hobson, 1971; Huebner and Chadwick, 2012a; Losey, 1972; Stummer et al., 2004); therefore, client colour patterns may serve as an interspecific signal. Here, we quantified cleaner shrimp visual capabilities to ask whether cleaner shrimp can reliably perceive the colours and patterns of conspecifics and/or clients.

We examined the visual physiology of three cleaner shrimp species: Lysmata amboinensis, Ancylomenes pedersoni and Urocaridella antonbruunii. To quantify spectral sensitivity and temporal resolution, we used electroretinography (ERG). ERG measures the physiological response of photoreceptors in situ, and is the method of choice for comparative studies, and for examining spectral sensitivities in animals whose sensitivities have not been previously quantified. Crustacean electroretinograms are monophasic; thus, the ERG response mirrors the response of the photoreceptors alone (Chapman and Lall, 1967; Goldsmith and Fernandez, 1968). To quantify spatial resolution, we used both morphological and behavioural methods. We then used those visual 


\section{List of symbols and abbreviations \\ $\mathrm{CFF}_{\max }$ maximum critical flicker fusion frequency \\ ERG electroretinography \\ I irradiance \\ $k \quad$ irradiance required to generate a response of $50 \% V_{\max }$ \\ $V \quad$ voltage \\ $V_{\max } \quad$ maximum voltage the eye is capable of producing, maximum electrical response created by the photoreceptors \\ $\alpha_{\min } \quad$ minimum resolvable angle, a measure of spatial resolution \\ $\Delta \Phi \quad$ interommatidial angle, the separation between adjacent ommatidial axes}

parameters to provide a general sense of how cleaner shrimp might view the patterns and colours of conspecifics and client fish.

These three cleaner shrimp species were selected because they are known fish cleaners (Becker et al., 2005; Chen and Huang, 2012; Côté, 2000), are colourful and finely patterned, operate cleaning stations with conspecifics, and live on shallow coral reefs (often $<20 \mathrm{~m}$ deep), which are spectrally broad, high light intensity environments. We assumed that cleaner shrimp, which display a variety of colours, have colour vision. Additionally, based on the high light intensity environment in which they live and their regular interactions with finely patterned conspecifics and client fish, we predicted that cleaner shrimp have high temporal resolution and fine spatial acuity that would allow them to perceive conspecific and client colour patterns over ecologically relevant distances.

\section{MATERIALS AND METHODS \\ Animals}

The three species used in this study were selected to represent species from different geographic locations and different genera that contain cleaner shrimp. Lysmata amboinensis (De Man 1888) is found throughout the Red Sea and tropical Indo-Pacific, operating cleaning stations in pairs (Fletcher et al., 1995). Ancylomenes pedersoni (Chace 1958) is found throughout the Caribbean living in association with anemone hosts, in groups of $2-17$, but occasionally more, individuals (Briones-Fourzán et al., 2012). Lastly, Urocaridella antonbruunii (A. J. Bruce 1967) inhabits reefs in the Indian Ocean, and lives in groups of at least 10 individuals (Chen and Huang, 2012).

Lysmata amboinensis and U. antonbruunii were purchased from Consistent-Sea Inc. (Gardena, CA, USA), and A. pedersoni from Dynasty Marine Associates, Inc. (Marathon, FL, USA). All individuals were wild-caught. ERG was performed at the Nova Southeastern University Oceanographic Center (Dania Beach, FL, USA). Animals were housed in aquaria using flow-through seawater drawn from an inlet to the Atlantic just off the coast of Dania Beach, FL, USA. Animals used for recording electroretinograms were kept in the dark, to keep them as dark-adapted as possible prior to use in experiments. During optomotor experiments, animals were housed in aquaria at Duke University (Durham, NC, USA) on a $12 \mathrm{~h}: 12 \mathrm{~h}$ light:dark cycle, and in artificial seawater made from Instant Ocean (United Pet Group, Blacksburg, VA, USA). Temperature was kept at approximately $23-24^{\circ} \mathrm{C}$, with salinity maintained between 28 and 31 ppt. Animals were fed one stick of Crab Cuisine (Hikari ${ }^{\circledR}, \mathrm{CA}$, USA) three times per week.

\section{Electrophysiology \\ Equipment}

Electroretinographic recording was used to measure spectral sensitivity $(N=4-8$ individuals/species) and temporal resolution
( $N=4-6$ individuals/species). During trials, a plastic post was attached to the dorsal surface of the animal using cyanoacrylate glue (Super Glue, Pacer Technology, Rancho Cucamonga, CA, USA), and the animal was suspended in seawater, which was maintained at room temperature (approximately $23^{\circ} \mathrm{C}$ ). The animal was placed such that its eyes were out of the water, but the rest of the body was submerged, leaving the pleopods free to move and circulate water.

A tungsten microelectrode (FHC Inc., Bowdoin, ME, USA) was inserted beneath the cornea of the left eye under dim red light (610 nm cut-off filter), which minimized light adaptation of the eye. This electrode was used to record electrical responses to light stimuli; a differential reference electrode was placed in the surrounding seawater, which was grounded by an $\mathrm{AgCl}$-coated wire. The eye was exposed to light stimuli, and $\mathrm{AC}$ recordings of the eye's response were amplified, digitized, and stored using a custom program written in LabView (National Instruments, Austin, TX, USA). Cut-off frequencies were $1 \mathrm{~Hz}$ and $15 \mathrm{KHz}$. We used a Spectral Products (Putnam, CT, USA) CM110 monochromator (175 W xenon lamp) to shine a stimulus light of controlled wavelength on the eye of the animal, via one branch of a bifurcated, randomized fibre light guide (EXFO, Quebec City, QC, Canada), which was placed so as to bathe the entire eye in a diffuse circle of light (rather than exposing the eye to a pinpoint source). Bathing the entire eye in light allowed as many ommatidia as possible to contribute to the response, and maximized the likelihood that we would be able to detect any secondary receptor types if present. A computer-controlled shutter (model VS25, Uniblitz, Rochester, NY, USA) set the stimulus duration. The irradiance of the stimulus light was dictated using a neutral density wheel controlled by the computer, and irradiance was calibrated in units of photons $\mathrm{cm}^{-2} \mathrm{~s}^{-1}$ for data analysis.

\section{Spectral sensitivity}

Following placement of the electrode, organisms were dark adapted for 1-2 $\mathrm{h}$, and then exposed to a standard flash of set wavelength and intensity (test flash). Once the response to test flashes had remained steady for half an hour, spectral sensitivity measurements were initiated using $100 \mathrm{~ms}$ flashes of monochromatic light; irradiance of the flash was adjusted until a criterion response was achieved (defined as the minimum response distinguishable from background noise, either 50 or $100 \mu \mathrm{V}$ ) at each wavelength. Animals were tested at $10 \mathrm{~nm}$ intervals from 350 to $610 \mathrm{~nm}$. Standard flashes of set wavelength and irradiance were presented to the animal approximately every five flashes, to ensure that the animal remained dark adapted throughout the experiment.

Once the criterion response had been reached at each wavelength, chromatic adaptation experiments were performed, wherein adapting light was presented to the eye via the second branch of the bifurcated light guide. The adapting light was a white light from a halogen lamp (LS-1, Ocean Optics, Inc., Dunedin, FL, USA), filtered with a $381 \mathrm{~nm}$ filter (full width at half maximum, FWHM $14 \mathrm{~nm}$ ), a $487 \mathrm{~nm}$ filter (FWHM $10 \mathrm{~nm}$ ) or a $550 \mathrm{~nm}$ longpass filter. The irradiance of the adapting light was adjusted with neutral density filters so that the irradiance required to obtain the criterion response at a given wavelength was at least one log unit brighter than when dark-adapted. Animals were chromatically adapted until standard flashes remained steady for $10 \mathrm{~min}$ (this usually occurred within $30 \mathrm{~min}$ of adaptation), before beginning spectral sensitivity measurements. Animals were then tested on the same series of wavelengths as above, including test flashes, until the criterion response was reached at each wavelength. For each individual, we 
recorded a dark-adapted spectrum and a chromatically adapted spectrum, then allowed the animal to dark-adapt once again, and recorded another chromatically adapted spectrum. We repeated this process with each individual shrimp for as many chromatic adaptations as was possible before the ERG degraded.

To visualize the spectral sensitivity data, we plotted the reciprocal of the irradiance (photons $\mathrm{cm}^{-2} \mathrm{~s}^{-1}$ ) required to produce the criterion response at each wavelength. We fitted absorbance curves to the spectral sensitivity data using visual pigment templates (Stavenga et al., 1993). To statistically compare dark-adapted and chromatically adapted curves within each species, we split the spectral sensitivity curves into four categories: UV (350-400 nm), blue (410-470 nm), SensMax (the region of maximum sensitivity, 480-540 nm) and red (550-610 nm) (following Cohen and Frank, 2007; Frank et al., 2009). For each individual, we calculated the mean inverse irradiance required to generate a criterion response to test flashes within each category. We then calculated ratios for UV: SensMax, blue:SensMax and red:SensMax for dark-adapted and chromatically adapted curves. For example, for each species, the UV:SensMax ratio for dark-adapted eyes was compared with the UV:SensMax ratio for each chromatic adaptation, and so on with blue:SensMax and red:SensMax. A Shapiro-Wilk test was used to determine whether data were normally distributed. As in Frank et al. (2009), to compare a given ratio between dark-adapted and each chromatic adaptation, we used a one-way ANOVA for parametric data sets and a Kruskal-Wallis test for non-parametric data sets (Zar, 1999). We used the Bonferroni method to correct for multiple comparisons, with $N=9$, because we performed nine ratio comparisons per species.

\section{Temporal resolution and V/logI}

We quantified temporal resolution using two metrics. First, we used the maximum critical flicker fusion frequency $\left(\mathrm{CFF}_{\max }\right)$ to quantify temporal resolution in both dark- and light-adapted conditions. The CFF is the highest stimulus rate for which the eye can still produce responses to each flash, but is dependent upon irradiance. $\mathrm{CFF}_{\max }$ is not dependent upon irradiance, and is reached when the eye cannot follow increasing rates of stimulus flashes, even with increases in irradiance. $\mathrm{CFF}_{\max }$ can be compared between species, and is thus well suited for comparative studies (see, for example, Cohen and Frank, 2006; Frank, 1999, 2000; McComb et al., 2013). In many photoreceptors, light adaptation shortens the response time, resulting in faster temporal dynamics and higher temporal resolution (Frank, 2003); to account for this, we calculated $\mathrm{CFF}_{\max }$ first in dark-adapted eyes (eye exposed only to the flickering stimulus light) and then in light-adapted eyes (eye bathed in adapting light of controlled wavelength and also exposed to the flickering stimulus light).

To measure dark-adapted $\mathrm{CFF}_{\max }$, animals were allowed to darkadapt until standard flashes remained constant in magnitude for $30 \mathrm{~min}$ (1-2 h depending on the individual). A $15 \mathrm{~Hz}$ flickering stimulus pulse $(510 \mathrm{~nm}$, with a $50 \%$ dark cycle, $50 \%$ light cycle) was presented to the eye for $2 \mathrm{~s}$. If the eye produced the same number of response peaks as the number of stimulus flashes in the pulse train, the animal was considered able to resolve a stimulus of that speed. The rate of the stimulus pulse was then increased until the animal could no longer produce responses to every flash in the pulse train. At this point, the irradiance of the stimulus light was increased by approximately half a log unit. When, after three increases in light irradiance, the eye could not produce responses to every flash in the pulse train, the $\mathrm{CFF}_{\max }$ was considered to have been achieved. In between each pulse train, the eye was allowed to dark-adapt, which was confirmed by monitoring the magnitude of the response to the standard flash that was given once a minute until the eye was darkadapted.

For light-adapted $\mathrm{CFF}_{\max }$, the eye was bathed in a white adapting light, and the flickering light stimulus was transmitted through the other end of the bifurcated light guide. The same protocol as for dark-adapted $\mathrm{CFF}_{\max }$ was followed, except that animals did not dark-adapt in between pulses. To statistically compare dark- and light-adapted $\mathrm{CFF}_{\max }$ both within and between species, we used a two-way ANOVA. Because the two-way ANOVA showed a significant interaction between light/dark and species $(P<0.001)$, we performed post hoc pair-wise tests, using one-way ANOVA for normally distributed data, and non-parametric Wilcoxon tests for data that were not normally distributed.

Following temporal resolution measurements, eyes were allowed to fully dark-adapt, and then $V / \log I$ curves were generated. $V / \log I$ curves plot an eye's electrical response (in V) versus the base 10 logarithm of the irradiance $(\log I)$ required to generate that response, and they indicate an eye's dynamic range, the range of irradiances over which a photoreceptor operates. $V / \log I$ curves allowed us to calculate $V_{\max }$, the maximum response that the eye is capable of generating at any irradiance, or the point at which the photoreceptor response is saturated. To generate $V / \log I$ curves, we used $100 \mathrm{~ms}$ stimuli of $490 \mathrm{~nm}$ monochromatic light (following Frank, 2003), increasing stimulus irradiance step-wise in half log unit steps. Darkadaptation between each stimulus was confirmed by monitoring the magnitude of the response to the standard flash. $V / \log I$ curves were fitted with the Zettler modification of the Naka-Rushton equation (Naka and Rushton, 1966a,b; Zettler, 1969) for the irradiance response function of photoreceptors (as in Frank, 2003). In several preparations, the maximum response was not achieved experimentally, but rather was calculated using the Naka-Rushton equation; in these cases, if the highest response recorded in the eye using ERG reached $90 \%$ of the calculated $V_{\max }$, those data were used in the analysis. Data were then normalized with respect to the peak response $\left(V_{\max }\right)$, and averaged across individuals to obtain a species average $V / \log I$ curve.

Also from $V / \log I$ curves, we calculated (1) $\log k$, with $k$ defined as the irradiance required to generate a response of $50 \% V_{\max }$, and a relative measure of an eye's sensitivity; and (2) response latency, defined as the time between stimulus onset and the beginning of the photoreceptor response, measured at $50 \% V_{\max }$, which is an indicator of the speed of transduction in the photoreceptors of each species.

\section{Spatial resolution}

We used two methods to quantify spatial resolution, as represented by the minimum resolvable angle $\left(\alpha_{\min }\right)$. Minimum resolvable angle is inversely related to spatial acuity, so smaller $\alpha_{\min }$ values indicate higher visual acuity. First, we used a morphological measure, the interommatidial angle $(\Delta \Phi)$, which is the separation between adjacent ommatidial axes ( $N=6$ individuals/species) and an indicator of the spatial fineness with which an image is sampled (Land and Nilsson, 2002). $\Delta \Phi$ can be estimated based on external eye morphology, in particular the radius of curvature of the eye and the average diameter of the eye facets. Second, we used a behavioural optomotor assay ( $N=5$ individuals/species), which estimates spatial resolution by measuring an individual's response to a rotating grating of black and white stripes.

For the morphological measure, we used a fluorescence microscope to photograph the eyes of six individuals per species. Using Image $\mathrm{J}$, we measured eye diameter in 10 facets per 
Table 1. Measures of eye radius and facet diameter, and morphological and behavioural estimates of $\alpha_{\min }$ in Lysmata amboinensis, Ancylomenes pedersoni and Urocaridella antonbruunii

\begin{tabular}{lcc}
\hline & Lysmata amboinensis & Ancylomenes pedersoni \\
\hline Radius of curvature $(\mathrm{mm})$ & $0.93 \pm 0.05(N=6)$ & $0.35 \pm 0.01(N=6)$ \\
Eye facet diameter $(\mu \mathrm{m})$ & $43 \pm 1.7(N=10)$ & $26 \pm 0.6(N=10)$ \\
Morphological $\alpha_{\min }(\mathrm{deg})$ & $5.6 \pm 0.4(N=6)$ & $8.2 \pm 0.4(N=6)$ \\
Behavioural $\alpha_{\min }(\mathrm{deg})$ & $9.9 \pm 0.3(N=5)$ & $8.3 \pm 0.1(N=5)$ \\
\hline
\end{tabular}

Sample size $(N)$ indicates the number of individuals for all measurements except eye facet diameter, for which it indicates the number of facets per individual. In the case of behavioural spatial resolution $\left(\alpha_{\mathrm{min}}\right)$, we used the single lowest value exhibited by a given individual across all trials and averaged those values to calculate species averages (see Materials and methods for details). Values are means \pm s.e.m.

individual, though facet diameter varied little across the eye (Table 1). For the same eyes, we estimated local curvature of the eye by fitting circles to images of the eyes (following Baldwin Fergus et al., 2015); because the eyes were not perfectly spherical, we fitted several circles to each eye image. Both facet diameter and radius of curvature were averaged across individual, and then across species. We then calculated $\Delta \Phi$ by dividing the facet diameter $(D)$ by the local radius of curvature of the eye $(R) . \alpha_{\min }$ was then calculated by multiplying $\Delta \Phi$ by two and converting to degrees (Land and Nilsson, 2002).

To behaviourally measure spatial resolution, we used the optomotor response, a stereotypical behaviour elicited by placing an animal inside a rotating drum lined with vertical black and white stripes. If an animal can resolve the stripes, it will rotate its body in the same direction as the drum rotation (McCann and MacGinitie, 1965; Reichardt, 1961). The thinnest stripe width at which an animal still turns in response to the rotating drum is the point beyond which behavioural responses did not reach a criterion response; two times that width (i.e. the width of one black and one white stripe together) can be interpreted as the limit of the animal's spatial resolution, or $\alpha_{\min }$.

During optomotor trials, an animal was placed inside a waterfilled $30 \mathrm{~cm}$ diameter cylindrical acrylic tank. The tank was then lowered into a drum that measured $56 \mathrm{~cm}$ in diameter, and which was lined with vertical black and white stripes. The drum and the striped stimuli were rotated from below using a computer-controlled stepper motor (STP-MTRH-23079, AutomationDirect, Atlanta, GA, USA). Stimuli were printed using a laser printer and covered a range of widths from 4.1 to $16.3 \mathrm{deg}$ at intervals of $0.4 \mathrm{deg}$. We also printed a control that was uniformly $50 \%$ grey. The optomotor tank was illuminated from directly overhead by an LED light panel (Fotodiox Pro LED 312-DS, Fotodiox Inc., IL, USA). Light was held constant at $5600 \mathrm{~K}$.

Spatial resolution varies with the brightness of ambient light, and marine animals regularly experience large changes in brightness. For animals that live at roughly $20-30 \mathrm{~m}$ depth, variation in sky condition and clarity of water can result in ambient brightness varying over 3-4 orders of magnitude (for example, a sunny day with clear water versus a cloudy day with murky water). For this reason, we performed the optomotor assay under three different lighting conditions: bright (light panel at full brightness), medium ( $10 \%$ of full brightness) and dark ( $0.01 \%$ of full brightness). These three lighting conditions, ranging over four orders of magnitude in irradiance, roughly approximate the range of brightness that is normally experienced by animals that live underwater.

Individuals were tested on each lighting condition once a week for four consecutive weeks. Prior to the beginning of the experiment, individuals were placed in the test arena for a $15 \mathrm{~min}$ acclimation period. Just before testing began, an $8 \mathrm{~cm}$ diameter acrylic cylinder was lowered around the animal to restrict its movements towards or away from the drum during testing, minimizing changes in distance between the animal's eyes and the rotating stimulus. We then rotated the drum at a constant speed of approximately 6 rotations/minute, an optimal speed determined in preliminary experiments. If an individual responded to the rotation by spinning in the direction of rotation, the drum was rotated in the opposite direction to ensure that movement was occurring in response to the stripes. If an animal successfully completed one half-rotation in each direction, we considered it a positive response. If no rotation occurred within $1 \mathrm{~min}$, we considered that a negative response. Stimuli were presented in random order, i.e. not consistently from narrower to wider or vice versa. The grey control was used once during each trial; over the course of 156 trials, no individuals responded to the control.

To describe the spatial resolution data, we used the median and interquartile range of $\alpha_{\mathrm{min}}$, because the data were not normally distributed. We used a non-parametric Kruskal-Wallis ANOVA to determine whether lighting condition significantly affected spatial resolution both within a species and across species. We also used the Wilcoxon rank sums method to compare the morphological estimate of best spatial resolution and the best spatial resolution found behaviourally, to determine whether morphologically predicted spatial resolution differed significantly from the best resolution observed behaviourally.

\section{Perception of conspecifics and client fish by cleaner shrimp}

To investigate perception of conspecifics and clients by cleaner shrimp, we used Fourier methods to create images that approximate how cleaners perceive visual scenes, based on our spectral sensitivity and spatial resolution data (following Baldwin and Johnsen, 2011; Baldwin Fergus et al., 2015). We began with colour images $(1024 \times 1024$ pixels $)$, and extracted one colour channel, resulting in a greyscale image. The Fourier transform of the greyscale image was then multiplied by a modulation transfer function (MTF) whose contrast at the maximum resolvable spatial frequency was less than $2 \%$, a value that was chosen because it approximates the minimum contrast threshold in organisms in bright light conditions (Douglas and Hawryshyn, 1990), and data regarding these specific species were lacking. We then applied an inverse Fourier transform to recover the image as it would appear to the cleaner shrimp eye. The end result was an image in which spatial information spanning angles less than $\alpha_{\min }$ measured behaviourally had been eliminated.

Actual size of each image was estimated based on size data for adults of each species, and distance to the viewer was held constant; thus, different images subtend different angles. In approximations of intraspecific appearance, the viewer was assumed to be $2.5 \mathrm{~cm}$ away from the subject, a short but ecologically relevant distance over which intraspecific signalling could occur between individuals at the same cleaning station. To model perception of clients, the viewer was assumed to be $10 \mathrm{~cm}$ away, a value based on published measures of cleaner-client interaction distance (Becker et al., 2005; Chapuis and Bshary, 2010). 


\section{RESULTS}

\section{Spectral sensitivity}

In all three species, dark-adapted spectral sensitivity curves (Fig. 1A) showed strong evidence for only one sensitivity peak, with peak sensitivity (mean \pm s.e.m.) of $518 \pm 5 \mathrm{~nm}$ (L. amboinensis), $518 \pm 2 \mathrm{~nm}$ (A. pedersoni) and $533 \pm 3 \mathrm{~nm}$ ( $U$. antonbruunii). However, dark-adapted curves also showed a shoulder between 420 and $450 \mathrm{~nm}$ in L. amboinensis and A. pedersoni, and a peak in the UV (around $350 \mathrm{~nm}$ ) in all three species. To explore whether these were due to additional photoreceptor classes, we performed several chromatic adaptations. Chromatic adaptations are a powerful method for revealing the presence of secondary sensitivity peaks, even those that result from uncommon photoreceptor types or low levels of expression of a certain pigment.

First, to account for the small shoulder between 420 and $450 \mathrm{~nm}$ in both L. amboinensis and A. pedersoni, we performed chromatic adaptations using a $487 \mathrm{~nm}$ (FWHM $10 \mathrm{~nm}$ ) filter and a $550 \mathrm{~nm}$ cutoff longpass filter (Fig. 1B,C). If this shoulder were due to a secondary photoreceptor class, it should be significantly higher under $550 \mathrm{~nm}$ adaptation than under $487 \mathrm{~nm}$ adaptation. However, if it were not due to a secondary photoreceptor class, then we would expect no differences to arise during chromatic adaptations, and thus

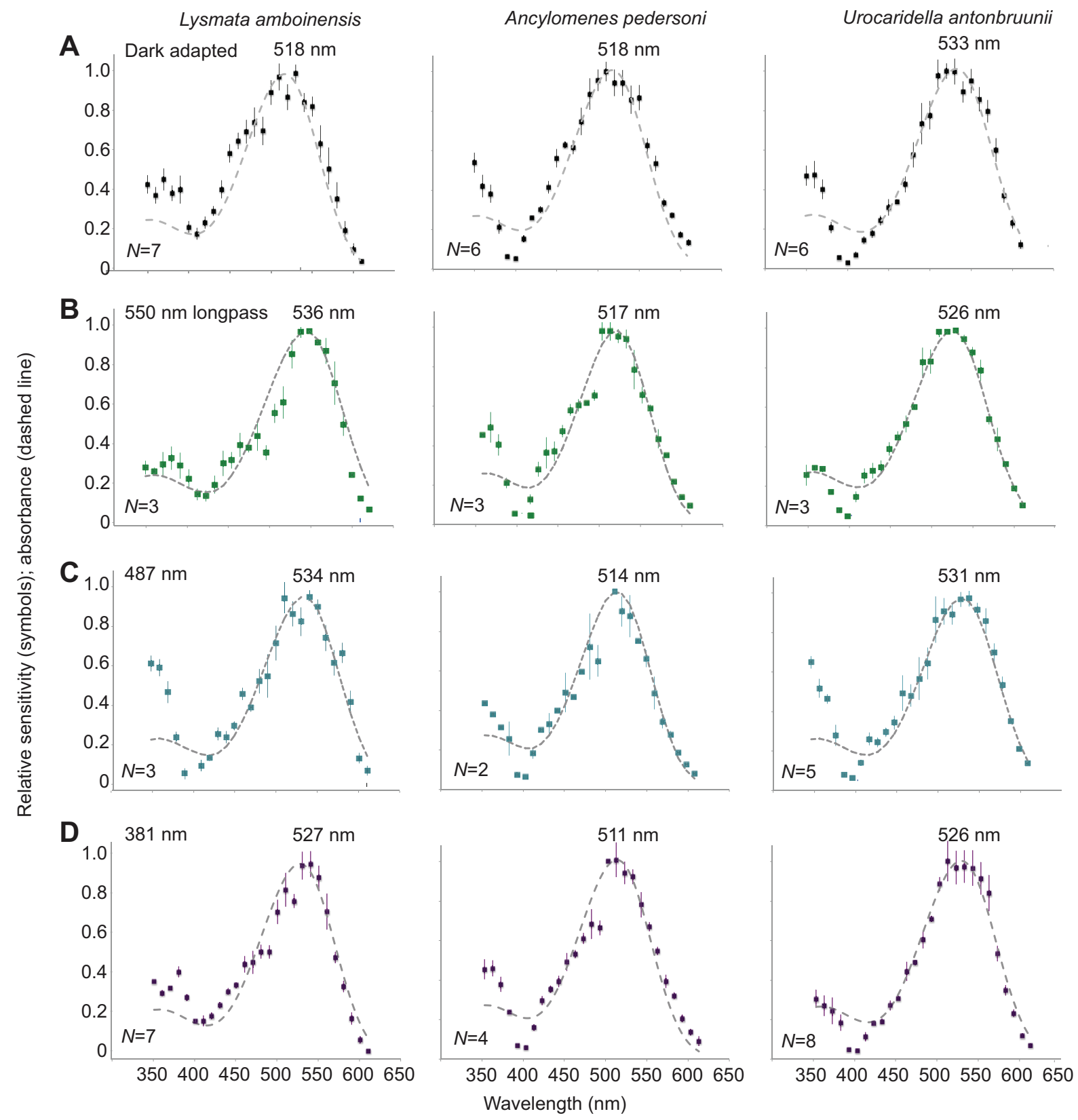

Fig. 1. Spectral sensitivity curves for Lysmata amboinensis, Ancylomenes pedersoni and Urocaridella antonbruunii. Cleaner shrimp were dark-adapted (A), and chromatically adapted using a $550 \mathrm{~nm}$ longpass filter (B), $487 \mathrm{~nm}$ filtered light (C) and $381 \mathrm{~nm}$ filtered light (D). All three species demonstrated only a single peak in each curve. Chromatic adaptation did not significantly shift the peak spectral sensitivity in any case (see Results for details of the statistical tests). Data points represent averaged, normalized spectral sensitivity data; dashed lines are best-fit absorbance curves. Numbers over each peak are peak spectral sensitivity. Sample size (individuals) is shown at the bottom left of each graph; differences in sample size arose because, for each individual, we recorded as many dark-adapted and chromatically adapted spectra as was possible before the response from the eye began to degrade, and the length of time that this allowed differed between individuals. Error bars represent s.e.m. 
Table 2. Dark- and light-adapted $\mathrm{CFF}_{\max }$, logk and latency of $50 \% V_{\max }$ response in three species of cleaner shrimp

\begin{tabular}{lll}
\hline & Lysmata amboinensis & Ancylomenes pedersoni \\
\hline CFF $_{\text {max }}$ : dark-adapted $(\mathrm{Hz})$ & $39 \pm 1.4(N=5)$ & $36 \pm 0.6(N=5)$ \\
$\mathrm{CFF}_{\text {max }}$ light-adapted $(\mathrm{Hz})$ & $42 \pm 0.8(N=5)$ & $48 \pm 0.9(N=5)$ \\
$k\left(\log\right.$ photons cm $\left.{ }^{-2} \mathrm{~s}^{-1}\right)$ & $13 \pm 0.3(N=4)$ & $13 \pm 0.2(N=5)$ \\
Response latency $(\mathrm{ms})$ & $28 \pm 2.6(N=4)$ & $25 \pm 2.4(N=5)$ \\
\hline
\end{tabular}

$\overline{C F F}_{\text {max }}$, maximum critical flicker fusion frequency; $k$, the irradiance required to generate a response of $50 \% V_{\text {max }} ; V_{\text {max }}$, maximum voltage response.

Sample size $(N)$ indicates the number of individuals per species. Values are means \pm s.e.m.

we would see no significant differences in the ratio of blue:SensMax between the $487 \mathrm{~nm}$ adaptation and $550 \mathrm{~nm}$ cut-off adaptation. As expected, in L. amboinensis, the average blue:SensMax ratio during $487 \mathrm{~nm}$ adaptation $(0.40 \pm 0.05)$ differed only slightly from the ratio during $550 \mathrm{~nm}$ adaptation $(0.38 \pm 0.03)$, and this difference was not significant (one-way ANOVA, $P=0.62$ ). In $A$. pedersoni, the difference in average blue:SensMax ratio during $487 \mathrm{~nm}$ adaptation $(0.57 \pm 0.18)$ compared with the $550 \mathrm{~nm}$ adaptation $(0.48 \pm 0.01)$ was greater, but this difference was still not significant (one-way ANOVA, $P=0.40$ ). Thus, these two chromatic adaptations provide evidence that the shoulder present between $420 \mathrm{~nm}$ and $450 \mathrm{~nm}$ is not due to a secondary photoreceptor class.
To investigate the peak in the UV wavelengths, we performed a chromatic adaptation using a $381 \mathrm{~nm}$ (FWHM $14 \mathrm{~nm}$ ) filter (Fig. 1D). We saw no significant differences in any of the ratios when comparing the $381 \mathrm{~nm}$ adaptation with any other chromatic adaptation (one-way ANOVA, every $P>0.06$ ). Thus, these results did not yield any support for the presence of a secondary photoreceptor class. The UV peak may be the $\beta$ peak of the primary visual pigment, although its magnitude is much greater than expected from the $\beta$ peak. Potentially, however, this peak may be due to a secondary, UV-sensitive photoreceptor class that we were unable to uncover using ERG.

Overall, the response waveforms during dark adaptation appeared virtually identical to those under chromatic adaptation to $381 \mathrm{~nm}$
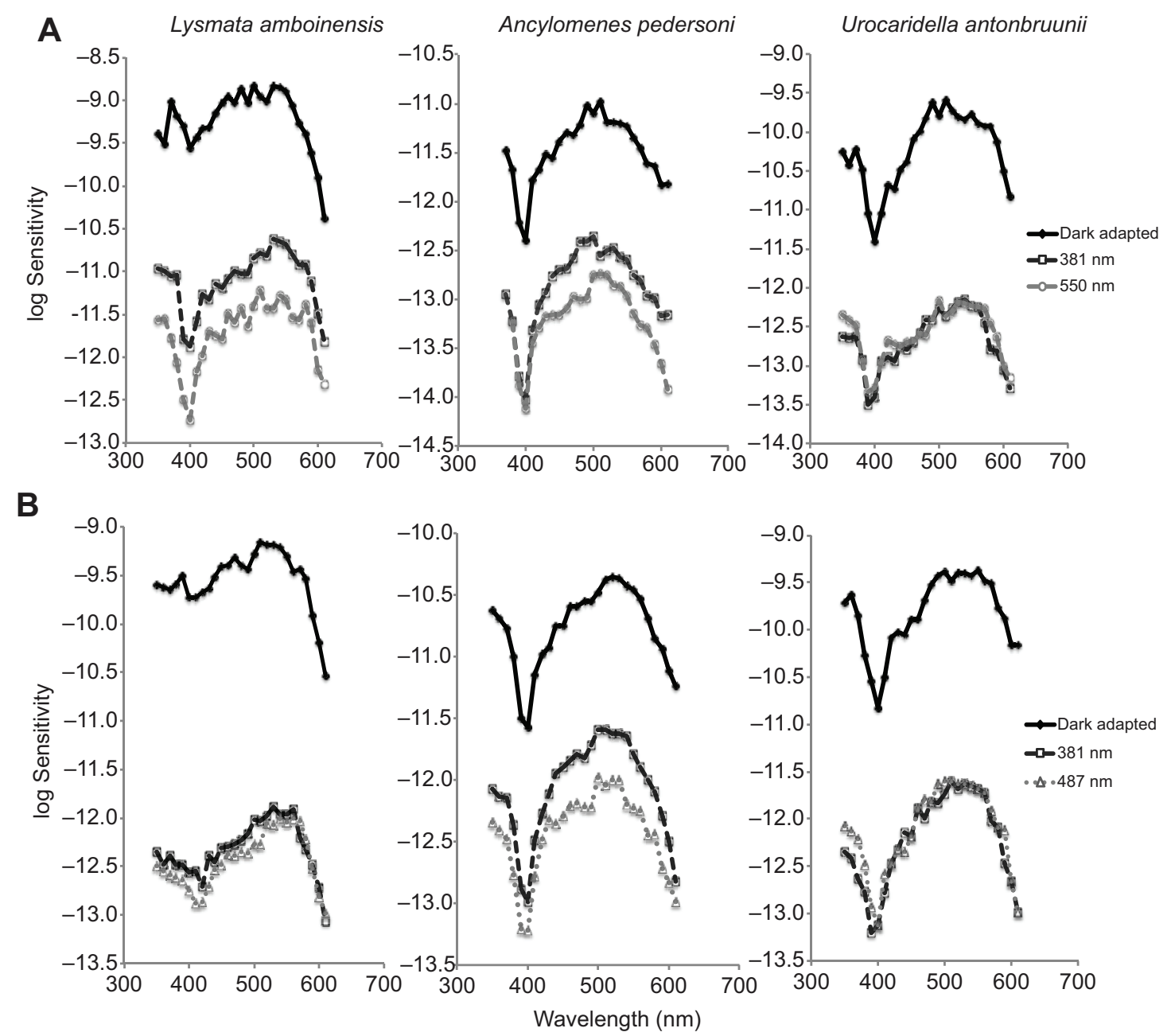

Fig. 2. Representative spectral sensitivity data for $L$. amboinensis, $A$. pedersoni and $U$. antonbruunii. Dark-adapted spectral sensitivity (solid lines) and chromatically adapted spectral sensitivity (dashed lines) were measured in preparations under (A) both $381 \mathrm{~nm}$ and $550 \mathrm{~nm}$ longpass chromatic adaptations and (B) both 381 and $487 \mathrm{~nm}$ chromatic adaptations. Each panel displays data from one preparation (i.e. specimen and electrode placement, $N=1$ individual for each panel). Similar results were found in all individuals for which multiple chromatic adaptations were performed. 
light, $486 \mathrm{~nm}$ light and $550 \mathrm{~nm}$ light. To compare dark-adapted spectral sensitivity curves with those under chromatic adaptation, we compared the UV:SensMax, blue:SensMax and red:SensMax ratios during chromatic adaptation with those from dark-adapted eyes, and found no significant differences in any ratio after Bonferroni correction (one-way ANOVA, every $P>0.10$ ). Thus, the lack of significant differences in sensitivity between dark-adaptation and any of the chromatic adaptations provides further evidence for the presence of only one photoreceptor class (Cohen and Frank, 2007; Frank et al., 2009). Fig. 2 shows results from individual preparations where spectral sensitivity curves from two chromatic adaptations, as well as dark-adapted curves, were recorded, and demonstrates that the shapes of the sensitivity functions were not preparation specific (i.e. were not dependent upon electrode placement).

Temporal resolution, sensitivity, response latency and V/logI All three species showed higher temporal resolution under light adaptation than dark adaptation (Table 2). Light adaptation resulted in a significant increase in $\mathrm{CFF}_{\text {max }}$ for both $A$. pedersoni $\left(F_{1,8}=120\right.$, $P<0.001)$ and $U$. antonbruunii $\left(F_{1,8}=9.66, \quad P=0.01\right)$; in L. amboinensis, the same trend was seen, but the difference between light- and dark-adapted $\mathrm{CFF}_{\max }$ was not significant $\left(F_{1,8}=3.27, P=0.11\right)$. Between-species comparisons showed that light-adapted $\mathrm{CFF}_{\max }$ differed significantly between $A$. pedersoni and $L$. amboinensis (Wilcoxon test; $Z=-2.54, P=0.01$ ), as well as between $A$. pedersoni and $U$. antonbruunii (Wilcoxon test; $Z=$ $-2.53, P=0.01)$. However, dark-adapted $\mathrm{CFF}_{\max }$ did not differ significantly between any species.

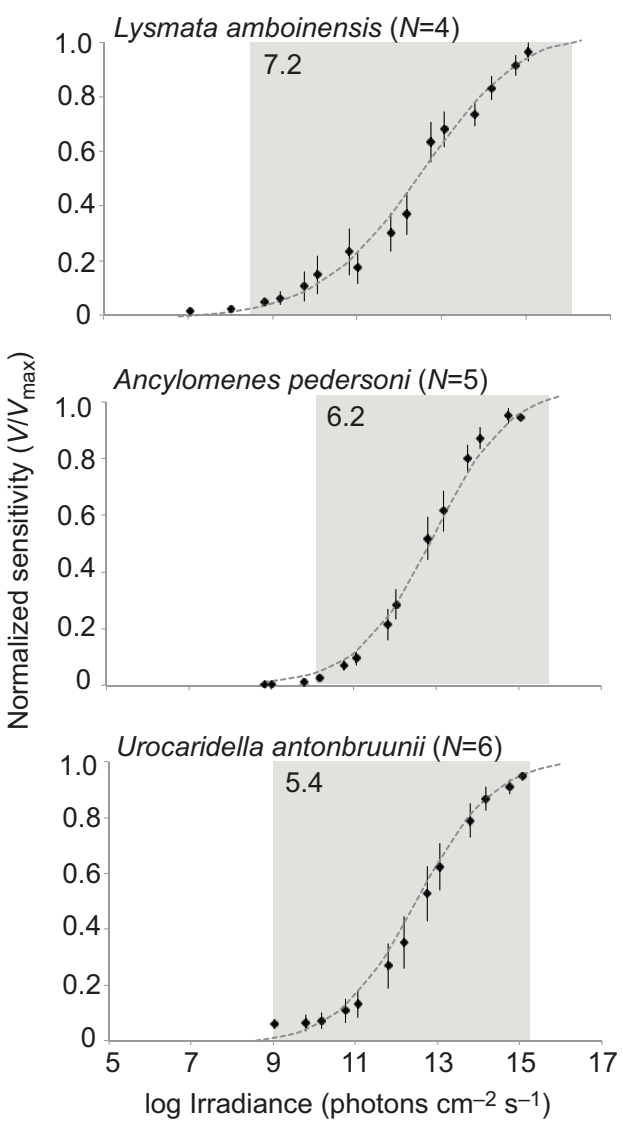

Fig. 3. V/log/ curves for $L$. amboinensis, A. pedersoni and $\boldsymbol{U}$. antonbruunii. The shaded boxes, and the numbers at the top left of each box, denote the dynamic range. Error bars are s.e.m.
All $V / \log I$ curves were sigmoidal in shape; dynamic ranges, $\log k$ and $V_{\max }$ are shown in Fig. 3. Response latency and overall sensitivity of the eye were similar for all species (Table 2). Both $\mathrm{CFF}_{\text {max }}$ and response latency are characteristics of a photoreceptor's temporal function, and previous studies have demonstrated that they are correlated (Frank, 2003). A Pearson correlation showed a good correlation between $\mathrm{CFF}_{\max }$ and response latency across species ( $N=15$ individuals; correlation coefficient $R=-0.59, P=0.046$ ), indicating that as response latency increases, flicker fusion rate decreases, as expected.

\section{Spatial resolution}

Measures of eye morphology (Table 1) estimated the theoretical best spatial resolution to be $5.6 \pm 1.0 \mathrm{deg}$ (L. amboinensis), $8.2 \pm 0.9 \mathrm{deg}(A$. pedersoni) and $7.2 \pm 0.7 \mathrm{deg}$ (U. antonbruunii), and thus predicted significant differences in spatial resolution between $L$. amboinensis and both $U$. antonbruunii (Wilcoxon test; $Z=2.16, P=0.03$ ) and $A$. pedersoni (Wilcoxon test; $Z=-2.64, P=0.008$ ). In each species, the finest spatial resolution measured behaviourally occurred at the brightest lighting condition (Fig. 4): $9.9 \pm 0.3 \mathrm{deg}$ (L. amboinensis), $8.3 \pm 0.1 \mathrm{deg}$ (A. pedersoni) and $11 \pm 0.50 \mathrm{deg}$ (U. antonbruunii). Behaviourally, A. pedersoni exhibited significantly finer resolution than both $L$. amboinensis (Wilcoxon test; $Z=2.19, P=0.03$ ) and $U$. antonbruunii (Wilcoxon test; $Z=2.35, P=0.02$ ).

Because spatial resolution can vary with the irradiance of ambient light, we performed the optomotor assay under three lighting conditions. As predicted, spatial resolution became coarser with a decrease in ambient light (Fig. 4). The difference between average $\alpha_{\min }$ at the brightest condition and the darkest condition was smallest in $A$. pedersoni ( $12 \%$ decrease, $Z=-2.19, P=0.03$ ), intermediate in L. amboinensis ( $29 \%$ decrease, $Z=-2.17, P=0.03$ ), and greatest in

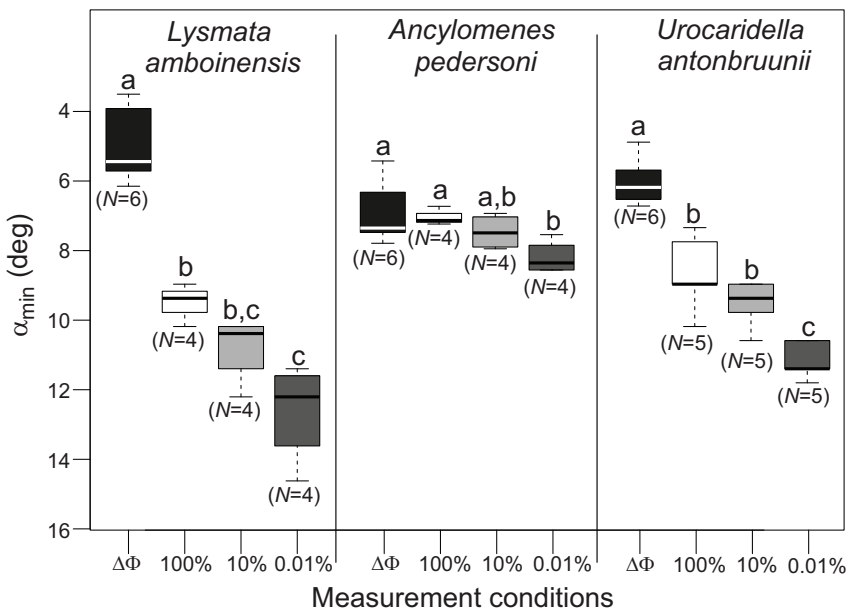

Fig. 4. Spatial resolution in L. amboinensis, $A$. pedersoni and $U$. antonbruunii. We estimated spatial resolution (represented by minimum resolvable angle, $\left.\alpha_{\mathrm{min}}\right)$ using interommatidial angle $(\Delta \phi$, black) and a behavioural optomotor assay under full intensity (white), 10\% intensity (light grey) and $0.01 \%$ intensity (dark grey) lighting conditions. Larger $\alpha_{\min }$ values translate to coarser spatial resolution; thus, in this graph, the finest spatial resolution is at the top. For each individual, we extracted the single lowest spatial value to which it responded during any trial and used those values to calculate species averages; the ranges indicated here thus illustrate the range of $\alpha_{\min }$ expressed across individuals (sample size indicated below each box). Boxes indicate the $25 \%$ and $75 \%$ quartiles, and the heavy black line indicates the median. Whiskers extend to the highest value that is within $1.5 \times$ the interquartile range. Letters above each box represent the results of withinspecies statistical comparisons; within a species, two boxplots that share a letter did not differ significantly (Wilcoxon paired tests; $P<0.05$ ). 
$U$. antonbruunii (30\% decrease, $Z=-2.53, P=0.01)$. As expected, decreased light levels resulted in decreased spatial resolution in all three species; thus, the ability of cleaner shrimp to resolve detail declines under darker conditions, such as deeper depths, darker times of day (e.g. dawn and dusk), cloudy days, or in murky water.

\section{Cleaner shrimp perception of conspecifics and clients}

Based on both our dark-adapted and chromatically adapted spectral sensitivity curves, we assumed monochromacy in our focal cleaner shrimp species. Thus, we modified achromatic photographs using our behavioural spatial resolution data to approximate perception of conspecifics and clients by cleaner shrimp. These photographs suggested that cleaner shrimp cannot resolve the fine spots and stripes that comprise conspecific appearance, even over distances of $2.5 \mathrm{~cm}$ (Fig. 5). Similarly, our results showed that cleaners can resolve the outlines of client fish, particularly against featureless backgrounds, but that cleaners cannot perceive the colours or finescale patterns of client fish (Fig. 6).

\section{DISCUSSION}

\section{Cleaner shrimp visual capabilities}

Here, we present the first study of spectral sensitivity, temporal resolution and spatial resolution in any species of cleaner shrimp. Using ERG, we found support for only a single photoreceptor class in the three cleaner shrimp we examined, with peak sensitivity between 515 and $535 \mathrm{~nm}$; if only one photoreceptor class is indeed present, then these cleaner shrimp are monochromatic, or colour blind (Fig. 1A). The majority of crustaceans whose spectral sensitivities have been studied ( primarily crabs, deep-sea shrimps or stomatopods) are either mono- or di-chromatic (reviewed in Marshall et al., 1999). However, previous research on crustacean spectral sensitivity has found that shallow-water coastal decapods are often dichromatic, with one photoreceptor maximally sensitive to blue-green wavelengths (480-540 nm), and a second UV/blue-sensitive photoreceptor with peak sensitivity near $400 \mathrm{~nm}$ (Cronin, 2006; Goldsmith and Fernandez, 1968; Johnson et al., 2002; Marshall et al., 1999, 2003), although monochromacy among shallow-water decapod crustaceans is not unprecedented (see Marshall et al., 1999 for a review).

We did observe two secondary shoulders or peaks in the darkadapted curves, one between 420 and $450 \mathrm{~nm}$ (in L. amboinensis and $A$. pedersoni) and one around $350 \mathrm{~nm}$, in all three species. However, chromatic adaptation experiments suggested that these were not indicative of secondary photoreceptor classes. Rather, the shoulder between 420 and $450 \mathrm{~nm}$ was probably due to experimental variation. The UV peak is likely to be the $\beta$ peak of the primary visual pigment, although the sensitivity values we recorded in the UV range are much higher than is expected from a $\beta$ peak. It is possible that the anomalously high values in the UV are indicative of a second, UV-sensitive photoreceptor class, though our chromatic adaptation experiments show evidence for only a single photoreceptor class (Fig. 1B-D). Crustaceans that are dichromatic tend to have UV/blue-sensitive photoreceptors (Marshall et al., 1999), suggesting that if our cleaner shrimp are in fact dichromatic, it is likely that their secondary spectral sensitivity peak would be in the UV. Other methods for examining spectral sensitivity (see below) could be used in future studies to search further for a UVsensitive photoreceptor class. Alternatively, the abnormally high sensitivity values recorded in the UV may be due to the presence of a UV-leaky screening pigment. Screening pigments are found in many crustaceans (for a discussion, see Douglas and Marshall, 1999), and in Gnathophausia ingens, previous work has shown that

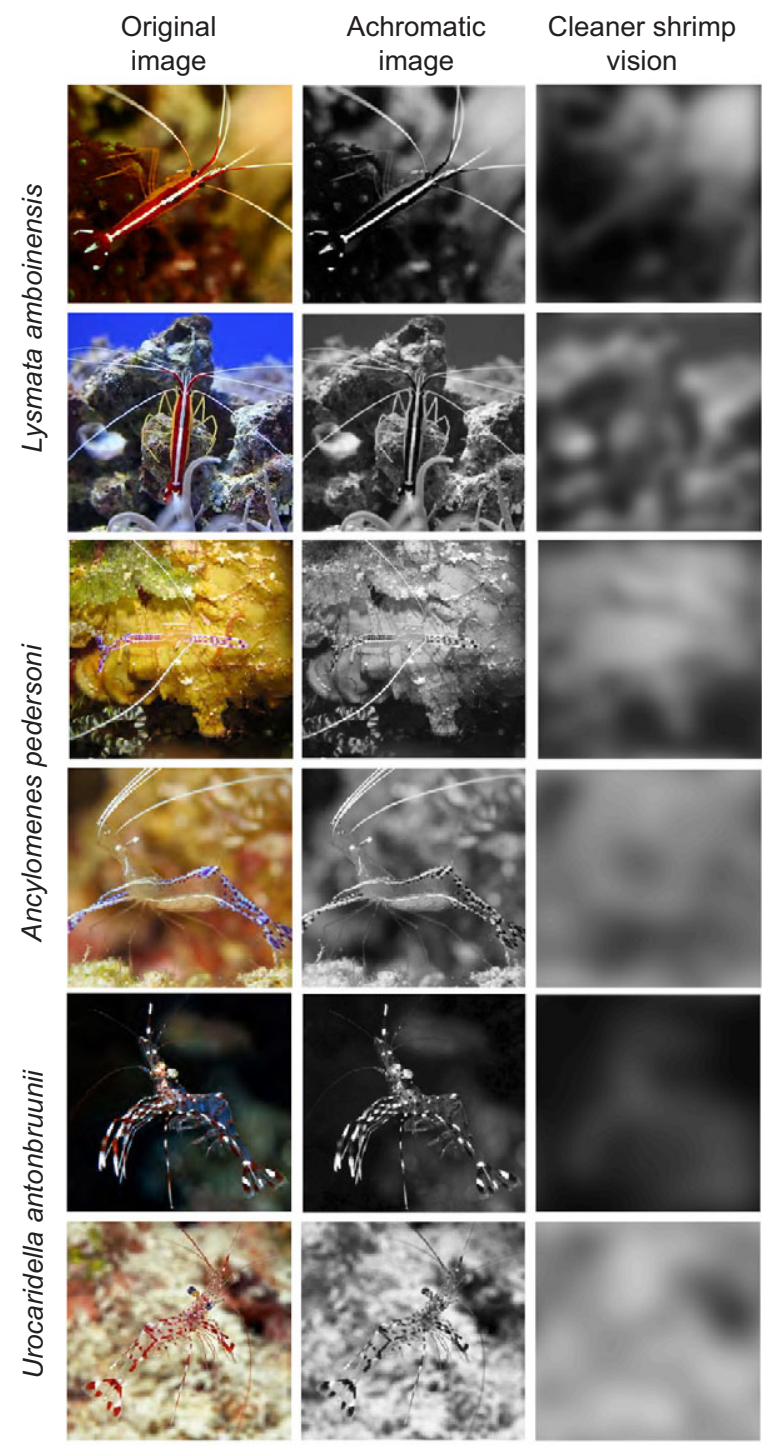

Fig. 5. Accounting for cleaner shrimp monochromatic vision and spatial resolution in intraspecific appearance. The three columns are the original image (left), an achromatic image created by isolating the green colour channel from the original image (middle), and simulated perception by a cleaner shrimp from a distance of $2.5 \mathrm{~cm}$ (right). Spatial resolution used to approximate cleaner shrimp vision was that measured using behavioural methods, under the brightest lighting condition. See Materials and methods for more details. Images attributed to: www.aquariumslife.com, Florence Charpin, Rokus Groeneveld and Ülar Tikk.

similar anomalous values recorded in the red end of the spectral sensitivity curves were due to the presence of red-leaky screening pigments (Frank et al., 2009).

Behaviourally quantified spatial acuity yielded $\alpha_{\min }$ between 8 and $11 \mathrm{deg}$ (Fig. 4), while morphological estimates yielded a theoretical best acuity estimate between 5 and $9 \mathrm{deg}$. A study of 96 species of decapod crustaceans found that $\Delta \phi$ ranged from 2.5 to $11.3 \mathrm{deg}$ (Dobson et al., 2014), putting cleaner shrimp on the higher end of known spatial resolution in decapods. However, compared with other published measures in the animal kingdom, cleaner shrimp spatial resolution is quite low, lower even than that found in sea snails (Littorina, $4.5 \mathrm{deg}$ ) or scallops (Pecten, $1.6 \mathrm{deg}$ ) (Land, 1981).

Lastly, we measured several indicators of temporal dynamics, including $\mathrm{CFF}_{\max }$ and response latency, as well as sensitivity, 


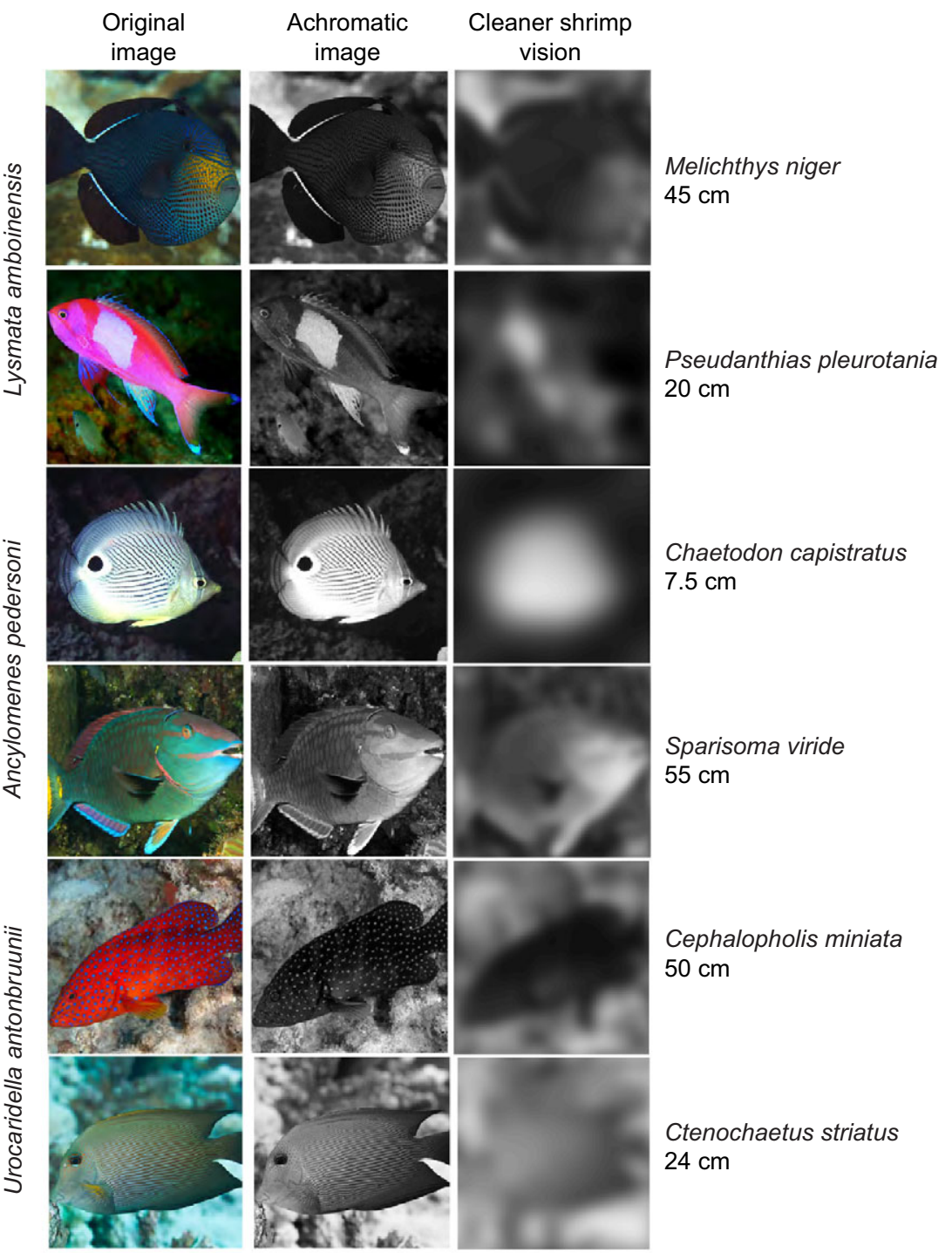

Fig. 6. Approximating client fish perception by cleaner shrimp. The three columns are the original image (left), an achromatic image created by isolating the green colour channel from the original image (middle), and simulated perception by a cleaner shrimp from a distance of $10 \mathrm{~cm}$ (right). Client species were chosen based on records of cleaner-client interactions from the literature (Becker et al., 2005; Chen and Huang, 2012; Wicksten, 1995, 2009), and to represent a range of client fish sizes. Adult size for each species is listed to the right of the photographs. Spatial resolution used to approximate cleaner shrimp vision was that measured using behavioural methods, under the brightest lighting condition. See Materials and methods for more details. Images attributed to: Mark Rosenstein, George Ryschkewitcsch, Brian Lasenby, Paddy Ryan and Bo Davidsson.

represented by $\log k$ (Table 2). Previous research on temporal resolution in crustaceans has for the most part focused on mesopelagic and deep-sea species (see Frank, 1999, 2000), so valid comparisons for values of $\mathrm{CFF}_{\max }$ between our cleaner shrimp and other species are difficult to make. However, a small number of studies have examined response latency and sensitivity in semiterrestrial and terrestrial crustaceans, which live in high-light environments and thus provide a more valid comparison with cleaner shrimp. In the fiddler crabs Uca thayeri and Uca pugilator, values of $\log k$ are 13.9 and 14.8 , thus only slightly higher than the values we observed here (reported in Cohen and Frank, 2006). The beach-dwelling amphipod Talorchestia longicornis shows only slightly lower values of $\log k$ (dark-adapted $11.89 \log$ photons $\mathrm{cm}^{-2} \mathrm{~s}^{-1}$ /light-adapted $11.97 \log$ photons $\mathrm{cm}^{-2} \mathrm{~s}^{-1}$ ) than cleaner shrimp, as well as slightly longer response latencies (darkadapted $30.3 \mathrm{~ms}$ /light-adapted $27.7 \mathrm{~ms}$ ) (Cohen et al., 2010). Thus, measures of sensitivity and response latency in our three species of cleaner shrimp were consistent with those of other studied crustaceans that live in a high-light environment.

\section{Potential functions of cleaner shrimp vision}

Using ERG, we found evidence for only a single photoreceptor class, which is suggestive of monochromacy, and our spatial resolution data show that cleaner shrimp have coarse vision. Thus, it is likely that they cannot resolve either the colours or intricate patterns that comprise their own appearances, even over short distances (Fig. 5). Similarly, cleaners cannot resolve fine-scale features on client fish (Fig. 6). However, our results do suggest that cleaner shrimp are capable of detecting large, looming stimuli - such as the outlines of client fish and changes in ambient light caused by approaching clients. This is supported by observations that cleaners respond with advertising motions when humans approach the aquaria where they are housed (E.M.C., personal observation). Additionally, one behavioural study has shown that cleaner shrimp will exhibit advertising behaviour towards large rectangular pieces of paper, suggesting that perhaps a looming visual stimulus, rather than a stimulus of particular shape, colour or detail, is the only cue necessary to initiate cleaning (Colin, 1972). Interestingly, predation on cleaner shrimp appears to be rare (summarized in Côté, 2000); thus, although a looming stimulus could also indicate the approach of a predator, it is likely that, on average, it is beneficial to cleaner shrimp to not flee from looming stimuli.

It has been suggested that any cleaning organism - from birds to fish to shrimp - should have good vision, as by definition cleaning organisms must be able to locate small ectoparasites living on other animals (Poulin and Grutter, 1996). Although ectoparasites vary greatly in size, studies of cleaner shrimp diets suggest that cleaners 
feed primarily on small ectoparasites approximately $0.1-2.5 \mathrm{~mm}$ in length (Becker and Grutter, 2004; McCammon et al., 2010). For an ectoparasite to be one 'pixel' across to a cleaner shrimp visual system with spatial resolution of 9 deg, the shrimp would have to be $15.8 \mathrm{~mm}$ away from a parasite $2.5 \mathrm{~mm}$ in length, but $0.63 \mathrm{~mm}$ away from a parasite $0.1 \mathrm{~mm}$ in length. Thus, though larger ectoparasites may be resolvable by the cleaner shrimp visual system, very small ones may not.

\section{The function of cleaner shrimp colour patterns}

We suggest that selection to resolve the colour patterns of conspecifics, for example during conspecific signalling, has not played a large role in the evolution of cleaner shrimp colour patterns. It may be, however, that selection on other viewers, for example client fish, to see cleaner shrimp has played a role in the evolution of cleaner shrimp appearance. Given that cleaner shrimp receive food from clients (Côté, 2000), it would be beneficial for them to be conspicuous to reef fish, and reef fish have both colour vision and good spatial acuity that may allow them to resolve cleaner shrimp colour patterns (Collin and Pettigrew, 1989; Losey et al., 1999b, 2003; Siebeck et al., 2008). There is evidence from certain reef fish that colour patterns can serve simultaneously as both a conspicuous signal and camouflage, depending on viewing distance and the background against which an animal is viewed (Marshall, 2000). Many cleaner species are symbiotic on host organisms, such as anemones or sponges, and thus different aspects of cleaner shrimp appearance may represent some combination of conspicuousness and host-specific camouflage, depending on signalling behaviour, body position, background and distance to viewer.

\section{Limits of this study}

Proving monochromacy is inherently difficult and, as discussed above, anomalously high sensitivity values in the UV could indicate the presence of a second, UV-sensitive pigment in cleaner shrimp. There are several methods that could potentially provide additional evidence for mono- or di-chromacy, including histology, genomics and microspectrophotometry (MSP).

Histologically, the presence of R8 cells can be suggestive of, but does not provide definitive evidence for, UV-sensitivity in crustaceans (Marshall et al., 1999). An organism may be UV sensitive without possessing an R8 cell (e.g. the isopod Ligia exotica; Hariyama et al., 1993), or R8 cells may be present but vestigial or small, and thus not functional (as in the eyes of deep-sea oplophorids; Frank and Widder, 1999).

Genetically, opsin gene sequences can help in determining an animal's spectral sensitivity. However, an expressed opsin does not necessarily have any functional significance in vision (for example, in the onycophoran Euperipatoides kanangrensis; Eriksson et al., 2013). Additionally, many crustaceans express more opsins than would be predicted based on photoreceptor physiology, as has been shown in stomatopods (Porter et al., 2013), brachyuran crabs (Rajkumar et al., 2010; Sakamoto et al., 1996), mysids (Frank et al., 2009), ostracods (Oakley and Huber, 2004) and branchipods (Kashiyama et al., 2009). Retinal transcriptomes, however, could be used to provide evidence for functional, expressed opsins.

Lastly, MSP can provide information on the absorption characteristics of visual pigments, and could potentially provide further evidence for the existence of a secondary photoreceptor class. However, MSP can have difficulty identifying pigments that exist in small quantities or are situated in small cells, and cannot measure the effects of pre-retinal filtering, self-screening and reflecting pigments (Goldsmith, 1978; Marshall et al., 1991). Thus, it should be noted that
MSP does not always give an accurate picture of the presence of visual pigments or their wavelength of peak sensitivity in situ. Ultimately, a combination of the above approaches, in addition to the ERG data presented here, may be necessary to draw firm conclusions regarding monochromacy in cleaner shrimp.

\section{Conclusions}

Whether cleaner shrimp are mono- or di-chromatic, they will be unable to perceive the rich diversity of colours found on conspecifics and clients that are so readily visible to human observers. Additionally, we show here that cleaner shrimp vision is quite coarse, and that low spatial resolution, rather than their colour vision capabilities, is likely to be the limiting factor on what they are able to view as signals. Ultimately, we can conclude that cleaner shrimp visual capabilities are not sufficient for them to view the colour patterns of conspecifics and client fish as signals, even over short distances. Future studies should consider whether cleaners use chemosensory, tactile or other sensory abilities in intraspecific communication, interacting with clients or locating ectoparasites. Additionally, how client fish perceive cleaner shrimp appearance is an avenue of study that may shed light on the selective pressures shaping the colour patterns of cleaner shrimp.

It is now well accepted among biologists that it is necessary to study the colour and luminance of visual signals from the perspective of relevant receivers (Bennett et al., 1994; Safran and Vitousek, 2008). We show here that the same is true of the spatial component of visual signals. This research underscores the importance of considering more than spectral sensitivity, for example by incorporating receiver-appropriate measures of spatial resolution, before drawing conclusions about the fitness consequences of any visual signal.

\section{Acknowledgements}

We thank two anonymous reviewers, Dr Jonathan Cohen, Dr Claire Spottiswoode, Dr Nicholas Brandley, Benjamin (Jay) Wheeler, Laura Bagge and Kate Thomas for comments on earlier versions of this manuscript.

\section{Competing interests}

The authors declare no competing or financial interests.

\section{Author contributions}

E.M.C. and T.M.F. designed and executed the electrophysiology experiments and analysed the data. E.M.C. conducted spatial resolution experiments, analysed the data and wrote the manuscript. T.M.F. wrote the custom software (to obtain the software, interested parties should contact the corresponding author). T.M.F. and S.J. provided intellectual input. S.J. produced images that modelled visual perception by cleaner shrimp, and revised the manuscript. All authors reviewed and corrected the manuscript.

\section{Funding}

E.M.C. was supported by the Department of Defense through the National Defense Science \& Engineering Graduate Fellowship (NDSEG) Program, and a Journal of Experimental Biology Travelling Fellowship (TF543).

\section{References}

Baldwin, J. and Johnsen, S. (2011). Effects of molting on the visual acuity of the blue crab, Callinectes sapidus. J. Exp. Biol. 214, 3055-3061.

Baldwin Fergus, J. L., Johnsen, S. and Osborn, K. J. (2015). A unique apposition compound eye in the mesopelagic hyperiid amphipod Paraphrnomia gracilis. Curr. Biol. 25, 1-9.

Bauer, R. T. (2006). Same sexual system but variable sociobiology: evolution of protandric simultaneous hermaphroditism in Lysmata shrimps. Integr. Comp. Biol. 46, 430-438.

Becker, J. H. and Grutter, A. S. (2004). Cleaner shrimp do clean. Coral Reefs 23 515-520.

Becker, J. H. A., Curtis, L. M. and Grutter, A. S. (2005). Cleaner shrimp use rocking dance to advertise cleaning service to clients. Curr. Biol. 15, 760-764.

Bennett, A. T. D., Cuthill, I. C. and Norris, K. (1994). Sexual selection and the mismeasure of color. Am. Nat. 144, 848-860. 
Briones-Fourzán, P., Pérez-Ortiz, M., Negrete-Soto, F., Barradas-Ortiz, C. and Lozano-Álvarez, E. (2012). Ecological traits of Caribbean sea anemones and symbiotic crustaceans. Mar. Ecol. Prog. Ser. 470, 55-68.

Bshary, R., Oliveira, R. F., Oliveira, T. S. F. and Canário, A. V. M. (2007). Do cleaning organisms reduce the stress response of client reef fish? Front. Zool. 4, 21.

Chapman, R. M. and Lall, A. B. (1967). Electroretinogram characteristics and the spectral mechanisms of the median ocellus and the lateral eye in Limulus polyphemus. J. Gen. Physiol. 50, 2267-2287.

Chapuis, L. and Bshary, R. (2010). Signalling by the cleaner shrimp Periclimenes longicarpus. Anim. Behav. 79, 645-647.

Chen, J. and Huang, H. (2012). A cleaning station composed of cleaner shrimp and high fish diversity in a coral reef in Kenting, Southern Taiwan. Collect. Res. 25, 41-51.

Cohen, J. H. and Frank, T. M. (2006). Visual physiology of the antarctic amphipod Abyssorchomene plebs. Biol. Bull. 211, 140-148.

Cohen, J. H. and Frank, T. M. (2007). Vision in the hyperiid amphipod Scina crassicornis. J. Mar. Biol. Assoc. UK 87, 1201-1206.

Cohen, J. H., Cronin, T. W., Lessios, N. and Forward, R. B. (2010). Visual physiology underlying orientation and diel behavior in the sand beach amphipod Talorchestia longicornis. J. Exp. Biol. 213, 3843-3851.

Colin, P. L. (1972). Model experiments on the releasing mechanism of cleaning behavior in the shrimp Periclimenes pedersoni. Atoll Res. Bull. 152, 2.

Collin, S. P. and Pettigrew, J. D. (1989). Quantitative comparison of the limits on visual spatial resolution set by the ganglion cell layer in twelve species of reef teleosts. Brain Behav. Evol. 34, 184-192.

Côté, I. M. (2000). Evolution and ecology of cleaning symbioses in the sea. Oceanogr. Mar. Biol. 38, 311-355.

Côté, I. M., Arnal, C. and Reynolds, J. D. (1998). Variation in posing behaviour among fish species visiting cleaning stations. J. Fish Biol. 53 Supp, 256-266

Cronin, T. W. (2006). Invertebrate vision in water. In Invertebrate Vision (ed. E. Warrant and D.-E. Nilsson), pp. 211-249. Cambridge: Cambridge University Press.

Cronin, T. W., Johnsen, S., Marshall, N. J. and Warrant, E. J. (2014). Visual Ecology. Princeton, NJ: Princeton University Press.

Dobson, N. C., De Grave, S. and Johnson, M. L. (2014). Linking eye design with host symbiont relationships in pontoniine shrimps (Crustacea, Decapoda Palaemonidae). PLoS ONE 9, e99505.

Douglas, R. H. and Hawryshyn, C. W. (1990). Behavioural studies of fish vision: an analysis of visual capabilities. In The Visual System of Fish (ed. R. H. Douglas and M. B. A. Djamgoz), pp. 373-418. Cambridge, UK: Chapman and Hall.

Douglas, R. H. and Marshall, N. H. (1999). A reivew of vertebrate and invertebrate ocular filters. In Adaptive Mechanisms in the Ecology of Vision (ed. S. N. Archer M. B. A. Djamgoz, E. R. Loew, J. C. Partridge and S. Vallerga), pp. 95-162. Great Britain: Kluwer Academic Publishers.

Eriksson, B. J., Fredman, D., Steiner, G. and Schmid, A. (2013). Characterisation and localisation of the opsin protein repertoire in the brain and retinas of a spider and an onychophoran. BMC Evol. Biol. 13, 186.

Fletcher, D. J., Kötter, I., Wunsch, M. and Yasir, I. (1995). Preliminary observations on the reproductive biology of ornamental cleaner prawns. Int. Zoo Yearb. 34, 73-77

Frank, T. M. (1999). Comparative study of temporal resolution in the visual systems of mesopelagic crustaceans. Biol. Bull. 196, 137.

Frank, T. M. (2000). Temporal resolution in mesopelagic crustaceans. Philos Trans. R. Soc. B. Biol. Sci. 355, 1195-1198.

Frank, T. M. (2003). Effects of light adaptation on the temporal resolution of deepsea crustaceans. Integr. Comp. Biol. 43, 559-570.

Frank, T. M. and Widder, E. A. (1999). Comparative study of the spectral sensitivities of mesopelagic crustaceans. J. Comp. Physiol. A Sens. Neural Behav. Physiol. 185, 255-265.

Frank, T. M., Porter, M. and Cronin, T. W. (2009). Spectral sensitivity, visual pigments and screening pigments in two life history stages of the ontogenetic migrator Gnathophausia ingens. J. Mar. Biol. Assoc. UK 89, 119-129.

Goldsmith, T. H. (1978). Effects of screening pigments on the spectral sensitivity of some crustacea with scotopic (superposition) eyes. Vision Res. 18, 475-482

Goldsmith, T. H. and Fernandez, H. R. (1968). Comparative studies of crustacean spectral sensitivity. Z. Vergl. Physiol. 60, 156-175.

Hariyama, T., Tsukahara, Y. and Meyer-Rochow, V. B. (1993). Spectra responses, including a UV-sensitive cell type, in the eye of the isopodLigia exotica. Naturwissenschaften 80, 233-235.

Hobson, E. S. (1971). Cleaning symbiosis among California inshore fishes. Fish Bull. 69, 491-523.

Huebner, L. K. and Chadwick, N. E. (2012a). Reef fishes use sea anemones as visual cues for cleaning interactions with shrimp. J. Exp. Mar. Biol. Ecol. 416-417, 237-242

Huebner, L. K. and Chadwick, N. E. (2012b). Patterns of cleaning behaviour on coral reef fish by the anemoneshrimp Ancylomenes pedersoni. J. Mar. Biol. Assoc. UK 92, 1557-1562.

Johnsen, S. (2006). Mathematical model of the visual abilities of sea turtles and pelagic fishes. In Sea Turtle and Pelagic Fish Sensory Biology: Developing
Techniques to Reduce Sea Turtle Bycatch in Longline Fisheries (ed. Y. Swimmer and R. Brill), pp. 18-23. U.S. Department of Commerce, NOAA Technical Memorandum, NOAA-TM-NMFS-PIFSC-7.

Johnson, M. L., Gaten, E. and Shelton, P. M. J. (2002). Spectral sensitivities of five marine decapod crustaceans and a review of spectral sensitivity variation in relation to habitat. J. Mar. Biol. Assoc. UK 82, 835-842.

Kashiyama, K., Seki, T., Numata, H. and Goto, S. G. (2009). Molecular characterization of visual pigments in Branchiopoda and the evolution of opsins in Arthropoda. Mol. Biol. Evol. 26, 299-311.

Land, M. F. (1981). Optics and vision in invertebrates. In Handbook of Sensory Physiology (ed. H. Autrum), pp. 471-592. Berlin: Springer.

Land, M. F. and Nilsson, D.-E. (2002). Animal Eyes. Oxford, UK: Oxford University Press.

Losey, G. S. (1972). The ecological importance of cleaning symbiosis. Copeia 1972 , 820-833.

Losey, G. S., Grutter, A. S., Rosenquist, G., Mahon, J. L. and Zamzow, J. P. (1999a). Cleaning symbiosis: a review. In Behaviour and Conservation of Littoral Fishes (ed. V. C. Almada, R. F. Oliveira and E. J. Gonçalves), pp. 379-395. Lisboa: ISPA

Losey, G. S., Cronin, T. W., Goldsmith, T. H., Hyde, D., Marshall, N. J. and McFarland, W. N. (1999b). The UV visual world of fishes: a review. J. Fish Biol. 54, 921-943

Losey, G. S., McFarland, W. N., Loew, E. R., Zamzow, J. P., Nelson, P. A. and Marshall, N. J. (2003). Visual biology of Hawaiian coral reef fishes. I. Ocular transmission and visual pigments. Copeia 2003, 433-454

Marshall, N. J. (2000). Communication and camouflage with the same 'bright' colours in reef fishes. Philos. Trans. R. Soc. B. Biol. Sci. 355, 1243-1248.

Marshall, N. J., Land, M. F., King, C. A. and Cronin, T. W. (1991). The compound eyes of mantis shrimps (Crustacea, Hoplocarida, Stomatopoda). II. Colour pigments in the eyes of stomatopod crustaceans: polychromatic vision by serial and lateral filtering. Philos. Trans. R. Soc. B Biol. Sci. 334, 57-84.

Marshall, J., Kent, J. and Cronin, T. W. (1999). Visual adaptations in crustaceans: spectral sensitivity in diverse habitats. In Adaptive Mechanisms in the Ecology of Vision (ed. S. N. Archer, M. B. A. Djamgoz, E. R. Loew, J. C. Partridge and S. Vallerga), pp. 285-327. Dordrecht, Netherlands: Kluwer Academic Publishers.

Marshall, N. J., Cronin, T. W. and Frank, T. M. (2003). Visual adaptations in crustaceans: chromatic, developmental, and temporal aspects. In Sensory Processing in Aquatic Environments (ed. S. P. Collin and N. J. Marshall), pp. 343-372. New York: Springer.

McCammon, A., Sikkel, P. C. and Nemeth, D. (2010). Effects of three Caribbean cleaner shrimps on ectoparasitic monogeneans in a semi-natural environment. Coral Reefs 29, 419-426.

McCann, G. D. and MacGinitie, G. F. (1965). Optomotor response studies of insect vision. Proc. R. Soc. B Biol. Sci. 163, 369-401.

McComb, D. M., Kajiura, S. M., Horodysky, A. Z. and Frank, T. M. (2013) Comparative visual function in predatory fishes from the Indian River Lagoon. Physiol. Biochem. Zool. 86, 285-297.

Naka, K. I. and Rushton, W. A. H. (1966a). S-Potentials from colour units in the retina of fish (Cyprinidae). J. Physiol. 185, 536-555.

Naka, K. I. and Rushton, W. A. H. (1966b). S-Potentials from luminosity units in the retina of fish (Cyprinidae). J. Physiol. 185, 587-599.

Oakley, T. H. and Huber, D. R. (2004). Differential expression of duplicated opsin genes in two eyetypes of ostracod crustaceans. J. Mol. Evol. 59, 239-249.

Osorio, D. and Vorobyev, M. (1996). Colour vision as an adaptation to frugivory in primates. Proc. R. Soc. B Biol. Sci. 263, 593-599.

Porter, M. L., Speiser, D. I., Zaharoff, A. K., Caldwell, R. L., Cronin, T. W. and Oakley, T. H. (2013). The evolution of complexity in the visual systems of stomatopods: insights from transcriptomics. Integr. Comp. Biol. 53, 39-49.

Poulin, R. and Grutter, A. S. (1996). Cleaning symbioses: proximate and adaptive explanations. Bioscience 46, 512-517.

Rajkumar, P., Rollmann, S. M., Cook, T. A. and Layne, J. E. (2010). Molecular evidence for color discrimination in the Atlantic sand fiddler crab, Uca pugilator. J. Exp. Biol. 213, 4240-4248.

Regan, B. C., Julliot, C., Simmen, B., Viénot, F., Charles-Dominique, P. and Mollon, J. D. (2001). Fruits, foliage and the evolution of primate colour vision. Philos. Trans. R. Soc. B Biol. Sci. 356, 229-283.

Reichardt, W. (1961). Sensory Communication. New York: John Wiley and Sons. Rufino, M. M. and Jones, D. A. (2001). Binary individual recognition in lysmata debelius (Decapoda: Hippolytidae) under laboratory conditions. J. Crust. Biol. 21, 388-392

Safran, R. J. and Vitousek, M. N. (2008). Evolutionary biology: arms races in the eye of the beholder. Curr. Biol. 18, R734-R736.

Sakamoto, K., Hisatomi, O., Tokunaga, F. and Eguchi, E. (1996). Two opsins from the compound eye of the crab Hemigrapsus sanguineus. J. Exp. Biol. 199, 441-450.

Sheehan, M. J., Jinn, J. and Tibbetts, E. A. (2014). Coevolution of visual signals and eye morphology in Polistes paper wasps. Biol. Lett. 10, 20140254.

Siebeck, U. E., Wallis, G. M. and Litherland, L. (2008). Colour vision in coral reef fish. J. Exp. Biol. 211, 354-360. 
Simoes, F. and Jones, D. A. (1999). Pair formation in tropical marine cleaner shrimp Lysmata debelius (Crustacea: Caridea). In Aquaculture 1999. USA: World Aquaculture Society.

Stavenga, D. G., Smits, R. P. and Hoenders, B. J. (1993). Simple exponential functions describing the absorbance bands of visual pigment spectra. Vision Res. 33, 1011-1017.

Stummer, L. E., Weller, J. A., Johnson, M. L. and Côté, I. M. (2004). Size and stripes: how fish clients recognize cleaners. Anim. Behav. 68, 145-150.
Wicksten, M. K. (1995). Associations of fishes and their cleaners on coral reefs of Bonaire, Netherlands Antilles. Copeia 1995, 477-481.

Wicksten, M. (2009). Interactions with fishes of five species of Lysmata (Decapoda, Caridea, Lysmatidae). Crustaceana 82, 1213-1223.

Zar, J. H. (1999). Biostatistical Analysis. Englewood Cliffs, NJ: Prentice-Hall, Inc. Zettler, F. (1969). Die Abhängigkeit des Ubertragungsverhaltens von Frequenz und Adaptationszustand; gemessen am einzelnen Lichtrezeptor von Calliphora erythrocephala. Z. Vergl. Physiol. 64, 432-449. 